\title{
The solvability conditions for the inverse eigenvalue problem of normal skew $J$-Hamiltonian matrices
}

Jia Zhao and Jieming Zhang*

"Correspondence:

zhangjmsw@126.com

Basic Course Department, Business

College of Shanxi University,

Taiyuan, P.R. China

\begin{abstract}
Let $J \in \mathbb{R}^{n \times n}$ be a normal matrix such that $\rho^{2}=-I_{n}$, where $I_{n}$ is an $n$-by- $n$ identity matrix. In (S. Gigola, L. Lebtahi, N. Thome in Appl. Math. Lett. 48:36-40, 2015) it was introduced that a matrix $A \in \mathbb{C}^{n \times n}$ is referred to as normal J-Hamiltonian if and only if $(A J)^{*}=A J$ and $A A^{*}=A^{*} A$. Furthermore, the necessary and sufficient conditions for the inverse eigenvalue problem of such matrices to be solvable were given. We present some alternative conditions to those given in the aforementioned paper for normal skew J-Hamiltonian matrices. By using Moore-Penrose generalized inverse and generalized singular value decomposition, the necessary and sufficient conditions of its solvability are obtained and a solvable general representation is presented.
\end{abstract}

MSC: 65F18; 15A51; 15A18; 15A12

Keywords: Inverse eigenvalue problem; Hamiltonian matrix; Normal matrix; Moore-Penrose generalized inverse; Generalized singular value decomposition

\section{Introduction}

In this paper, we mainly discuss the following partially described inverse eigenvalue problem which is considered in linear manifold.

Problem 1 Given the partial eigeninformation $Y=\left(y_{1}, y_{2}, \ldots, y_{m}\right) \in \mathbb{C}^{n \times m}$ and $\Lambda=$ $\operatorname{diag}\left(\lambda_{1}, \lambda_{2}, \ldots, \lambda_{m}\right) \in \mathbb{C}^{m \times m}$, consider the set

$$
\mathcal{M}(Y, \Lambda):=\{A \in \Omega \mid A Y=Y \Lambda\}
$$

of matrices $A$ maintaining the eigeninformation, where $\Omega$ is the set of certain $n$-by- $n$ structured matrices.

The above problem usually appears in the design and modification of mass-spring systems, dynamic structures, Hopfield neural networks, vibration in mechanic, civil engineering, and aviation [2-4]. Furthermore, the inverse eigenvalue problems involving Hamiltonian matrices have drawn considerable interest. For example, Zhang et al. [5] solved the inverse eigenvalue problem of Hermitian and generalized Hamiltonian matrices. Then Bai [6] settled the case of Hermitian and generalized skew-Hamiltonian matrices. Xie et al. [7] resolved the case of symmetric skew-Hamiltonian matrices. Qian

(c) The Author(s) 2018. This article is distributed under the terms of the Creative Commons Attribution 4.0 International License (http://creativecommons.org/licenses/by/4.0/), which permits unrestricted use, distribution, and reproduction in any medium, provided you give appropriate credit to the original author(s) and the source, provide a link to the Creative Commons license, and indicate if changes were made. 
and Tan [8] also considered the cases of Hermitian and generalized Hamiltonian/skewHamiltonian matrices from different perspectives. But the Hamiltonian matrices they considered are the special cases of the following normal $J$-Hamiltonian matrices and normal skew $J$-Hamiltonian matrices.

In the following, let $I_{n}$ be the $n \times n$ identity matrix.

Definition 1 ([1]) Given a normal matrix $J \in \mathbb{R}^{n \times n}$ with $J^{2}=-I_{n}$. A matrix $A \in \mathbb{C}^{n \times n}$ is referred to as normal $J$-Hamiltonian if and only if $(A J)^{*}=A J$ and $A A^{*}=A^{*} A$.

Definition 2 Let $J \in \mathbb{R}^{n \times n}$ be a normal matrix such that $J^{2}=-I_{n}$. $A$ matrix $A \in \mathbb{C}^{n \times n}$ is called normal skew $J$-Hamiltonian if and only if $(A J)^{*}=-A J$ and $A A^{*}=A^{*} A$. The set of all $n \times n$ normal skew $J$-Hamiltonian matrices is denoted by $\mathcal{N} \mathcal{S}^{n \times n}(J)$.

In the above definitions, $A^{*}$ signifies the conjugate transpose of a matrix $A \in \mathbb{C}^{n \times n}$. It is obvious that $J$ is a real orthogonal skew-symmetric matrix, i.e., $J=-J^{T}=-J^{-1}$. This indicates that $n=2 k, k \in \mathbb{N}$. The above Hamiltonian matrices are also of importance in several engineering areas such as optimal quadratic linear control, $H_{\infty}$ optimization, and the related problem of solving Riccati algebraic equations [9].

Recently, Gigola et al. [1] solved Problem 1 for normal $J$-Hamiltonian matrices. In this paper, we present a set of alternative conditions assuring the solvability of the problem that involves skew normal $J$-Hamiltonian matrices. In order to present more simple conditions to be verified, we mainly use Sun's [10] and Penrose's [11] results and the generalized singular value decomposition to solve Problem 1 when the set $\Omega=\mathcal{N} \mathcal{S}^{n \times n}(J)$. A similar technique may be used to solve the inverse eigenvalue problem for normal J-Hamiltonian matrices.

\section{Preliminaries}

Throughout this paper, we denote by $\operatorname{rank}(A)$ and $A^{\dagger}$ the rank and Moore-Penrose generalized inverse of a matrix $A \in \mathbb{C}^{n \times m}$, respectively. $I_{n}, \mathbf{0}$, and $\mathrm{i}=\sqrt{-1}$ respectively signify the identity matrix of order $n$, a zero matrix or a vector with appropriate size, and the imaginary unit. Moreover, for any matrix $A \in \mathbb{C}^{n \times m}, \mathscr{R}_{A}=I_{n}-A A^{\dagger}$ and $\mathscr{L}_{A}=I_{m}-A^{\dagger} A$ signify specified orthogonal projectors.

Firstly, we consider the structure of the normal skew $J$-Hamiltonian matrices.

Lemma 1 Let $J \in \mathbb{R}^{n \times n}$ be a normal matrix such that $J^{2}=-I_{n}$. Then a matrix $A \in \mathbb{C}^{n \times n}$ is normal skew J-Hamiltonian if and only if

$$
A=U\left[\begin{array}{cc}
A_{11} & A_{12} \\
-A_{12}^{*} & A_{22}
\end{array}\right] U^{*},
$$

where $n=2 k, k \in \mathbb{N}, A_{11}=A_{11}^{*} \in \mathbb{C}^{k \times k}, A_{22}=A_{22}^{*} \in \mathbb{C}^{k \times k}, A_{11} A_{12}=A_{12} A_{22}$, and $U \in \mathbb{C}^{n \times n}$ is a unitary matrix such that

$$
U^{*} U U=\left[\begin{array}{cc}
\mathrm{i} I_{k} & \mathbf{0} \\
\mathbf{0} & -\mathrm{i} I_{k}
\end{array}\right] .
$$


Proof Because $J \in \mathbb{R}^{n \times n}$ is a normal matrix and $J^{2}=-I_{n}$, then $J$ is a real orthogonal skewsymmetric matrix. Therefore, there exists a unitary matrix $U \in \mathbb{C}^{n \times n}$ such that (2.2) holds, where $n=2 k, k \in \mathbb{N}$.

Then partition $U^{*} A U$ conforms with (2.2) as

$$
U^{*} A U=\left[\begin{array}{ll}
A_{11} & A_{12} \\
A_{21} & A_{22}
\end{array}\right] .
$$

From Definition 2, we know that $J A J=-A^{*}$. It follows that

$$
\left[\begin{array}{cc}
-A_{11} & A_{12} \\
A_{21} & -A_{22}
\end{array}\right]=-\left[\begin{array}{cc}
A_{11}^{*} & A_{21}^{*} \\
A_{12}^{*} & A_{22}^{*}
\end{array}\right] .
$$

Thus we have

$$
A_{11}=A_{11}^{*}, \quad A_{22}=A_{22}^{*}, \quad A_{12}=-A_{21}^{*} \text {. }
$$

Because $A A^{*}=A^{*} A$, then from (2.3) and (2.4) we have $A_{11} A_{12}=A_{12} A_{22}$. Therefore, (2.1) holds.

Then we introduce the following results to solve Problem 1 later on.

Lemma 2 (Sun [10]) Let $A_{1}, B_{1} \in \mathbb{C}^{n \times m}$ be given. The linear matrix equation $X_{1} A_{1}=B_{1}$ has a Hermitian solution $X_{1} \in \mathbb{C}^{n \times n}$ if and only if

$$
B_{1} \mathscr{L}_{A_{1}}=0, \quad A_{1}^{*} B_{1}=B_{1}^{*} A_{1} .
$$

In this case, the general solution can be expressed as

$$
X_{1}=B_{1} A_{1}^{\dagger}+\left(B_{1} A_{1}^{\dagger}\right)^{*}-\frac{1}{2}\left(A_{1}^{\dagger}\right)^{*}\left(A_{1}^{*} B_{1}+B_{1}^{*} A_{1}\right) A_{1}^{\dagger}+\mathscr{R}_{A_{1}} R_{1} \mathscr{R}_{A_{1}}
$$

where $R_{1} \in \mathbb{C}^{n \times n}$ is an arbitrary Hermitian matrix.

In this lemma, the general solution can be also expressed as

$$
X_{1}=B_{1} A_{1}^{\dagger}+\left(B_{1} A_{1}^{\dagger}\right)^{*} \mathscr{R}_{A_{1}}+\mathscr{R}_{A_{1}} R_{1} \mathscr{R}_{A_{1}} .
$$

The following lemma is taken from [11], see Corollary 2 in [11].

Lemma 3 (Penrose [11]) Let $A_{2} \in \mathbb{C}^{n \times m}, C_{2} \in \mathbb{C}^{n \times p}, B_{2} \in \mathbb{C}^{p \times q}$, and $D_{2} \in \mathbb{C}^{m \times q}$ be given. The pair of matrix equations $A_{2} X_{2}=C_{2}, X_{2} B_{2}=D_{2}$ has a solution $X_{2} \in \mathbb{C}^{m \times p}$ if and only if

$$
\mathscr{R}_{A_{2}} C_{2}=0, \quad D_{2} \mathscr{L}_{B_{2}}=0, \quad A_{2} D_{2}=C_{2} B_{2}
$$

Moreover, the general solution can be expressed as

$$
X_{2}=A_{2}^{\dagger} C_{2}+\mathscr{L}_{A_{2}} D_{2} B_{2}^{\dagger}+\mathscr{L}_{A_{2}} R_{2} \mathscr{R}_{B_{2}},
$$

where $R_{2} \in \mathbb{C}^{m \times p}$ is an arbitrary matrix. 


\section{Solvability conditions and general solution of Problem 1}

Given a normal matrix $J \in \mathbb{R}^{n \times n}$ with $J^{2}=-I_{n}$, let $Y \in \mathbb{C}^{n \times m}$ and $\Lambda \in \mathbb{C}^{m \times m}$ be given in Problem 1. In order to solve this problem for the case of normal skew $J$-Hamiltonian matrices, we need to obtain the normal skew $J$-Hamiltonian solution of the linear matrix equation

$$
A Y=Y \Lambda
$$

If equation (3.1) is consistent, then the set $\mathcal{M}(Y, \Lambda)$ is nonempty. By Lemma 1, equation (3.1) is equivalent to the following:

$$
\left[\begin{array}{cc}
A_{11} & A_{12} \\
-A_{12}^{*} & A_{22}
\end{array}\right] U^{*} Y=U^{*} Y \Lambda .
$$

Let

$$
U^{*} Y=\left[\begin{array}{l}
Y_{1} \\
Y_{2}
\end{array}\right], \quad Y_{1} \in \mathbb{C}^{k \times m}, Y_{2} \in \mathbb{C}^{k \times m}
$$

Then (3.2) can be rewritten as follows:

$$
\left\{\begin{array}{l}
A_{11} Y_{1}+A_{12} Y_{2}=Y_{1} \Lambda \\
-A_{12}^{*} Y_{1}+A_{22} Y_{2}=Y_{2} \Lambda
\end{array}\right.
$$

Thus we have

$$
\left\{\begin{array}{l}
A_{12} Y_{2}=Y_{1} \Lambda-A_{11} Y_{1} \\
Y_{1}^{*} A_{12}=Y_{2}^{*} A_{22}-\Lambda^{*} Y_{2}^{*}
\end{array}\right.
$$

By Lemma 3, the above system of matrix equations has a solution $A_{12} \in \mathbb{C}^{k \times k}$ if and only if

$$
\left\{\begin{array}{l}
A_{11} Y_{1} \mathscr{L}_{Y_{2}}=Y_{1} \Lambda \mathscr{L}_{Y_{2}}, \\
A_{22} Y_{2} \mathscr{L}_{Y_{1}}=Y_{2} \Lambda \mathscr{L}_{Y_{1}}, \quad \text { where } \mathscr{L}_{Y_{1}}=I_{k}-Y_{1} Y_{1}^{*}, \\
Y_{1}^{*} A_{11} Y_{1}+Y_{2}^{*} A_{22} Y_{2}=Y_{1}^{*} Y_{1} \Lambda+\Lambda^{*} Y_{2}^{*} Y_{2}
\end{array}\right.
$$

Then, by Lemma 2, the first equation in (3.5) has a Hermitian solution $A_{11} \in \mathbb{C}^{k \times k}$ if and only if

$$
Y_{1} \Lambda \mathscr{L}_{Y_{2}} \mathscr{L}_{Y_{1} \mathscr{L}_{Y_{2}}}=0, \quad \mathscr{L}_{Y_{2}}\left(Y_{1}^{*} Y_{1} \Lambda-\Lambda^{*} Y_{1}^{*} Y_{1}\right) \mathscr{L}_{Y_{2}}=0
$$

In this case, the general solution is

$$
A_{11}=Y_{1} \Lambda \mathscr{L}_{Y_{2}}\left(Y_{1} \mathscr{L}_{Y_{2}}\right)^{\dagger}+\left(\mathscr{L}_{Y_{2}} Y_{1}^{*}\right)^{\dagger} \mathscr{L}_{Y_{2}} \Lambda^{*} Y_{1}^{*} \mathscr{R}_{Y_{1}} \mathscr{L}_{Y_{2}}+\mathscr{R}_{Y_{1}} \mathscr{L}_{Y_{2}} S_{1} \mathscr{R}_{Y_{1} \mathscr{L}_{Y_{2}}}
$$

where $S_{1} \in \mathbb{C}^{k \times k}$ is an arbitrary Hermitian matrix. 
Similarly, by Lemma 2 , the second equation in (3.5) has a Hermitian solution $A_{22} \in \mathbb{C}^{k \times k}$ if and only if

$$
Y_{2} \Lambda \mathscr{L}_{Y_{1}} \mathscr{L}_{Y_{2} \mathscr{L}_{Y_{1}}}=0, \quad \mathscr{L}_{Y_{1}}\left(Y_{2}^{*} Y_{2} \Lambda-\Lambda^{*} Y_{2}^{*} Y_{2}\right) \mathscr{L}_{Y_{1}}=0
$$

In this case, the general solution is

$$
A_{22}=Y_{2} \Lambda \mathscr{L}_{Y_{1}}\left(Y_{2} \mathscr{L}_{Y_{1}}\right)^{\dagger}+\left(\mathscr{L}_{Y_{1}} Y_{2}^{*}\right)^{\dagger} \mathscr{L}_{Y_{1}} \Lambda^{*} Y_{2}^{*} \mathscr{R}_{Y_{2}} \mathscr{L}_{Y_{1}}+\mathscr{R}_{Y_{2}} \mathscr{L}_{Y_{1}} S_{2} \mathscr{R}_{Y_{2} \mathscr{L}_{Y_{1}}},
$$

where $S_{2} \in \mathbb{C}^{k \times k}$ is an arbitrary Hermitian matrix. Let

$$
\begin{aligned}
G= & Y_{1}^{*} Y_{1} \Lambda+\Lambda^{*} Y_{2}^{*} Y_{2}-Y_{1}^{*}\left[Y_{1} \Lambda \mathscr{L}_{Y_{2}}\left(Y_{1} \mathscr{L}_{Y_{2}}\right)^{\dagger}+\left(\mathscr{L}_{Y_{2}} Y_{1}^{*}\right)^{\dagger} \mathscr{L}_{Y_{2}} \Lambda^{*} Y_{1}^{*} \mathscr{R}_{Y_{1}} \mathscr{L}_{Y_{2}}\right] Y_{1} \\
& -Y_{2}^{*}\left[Y_{2} \Lambda \mathscr{L}_{Y_{1}}\left(Y_{2} \mathscr{L}_{Y_{1}}\right)^{\dagger}+\left(\mathscr{L}_{Y_{1}} Y_{2}^{*}\right)^{\dagger} \mathscr{L}_{Y_{1}} \Lambda^{*} Y_{2}^{*} \mathscr{R}_{Y_{2}} \mathscr{L}_{Y_{1}}\right] Y_{2} .
\end{aligned}
$$

From (3.5), (3.7), and (3.9) we know that $G=G^{*}$ is equivalent to

$$
Y_{1}^{*} Y_{1} \Lambda+\Lambda^{*} Y_{2}^{*} Y_{2}=\Lambda^{*} Y_{1}^{*} Y_{1}+Y_{2}^{*} Y_{2} \Lambda
$$

Then substituting (3.7) and (3.9) into the third equation in (3.5) yields

$$
Y_{1}^{*} \mathscr{R}_{Y_{1} \mathscr{L}_{Y_{2}}} S_{1} \mathscr{R}_{Y_{1} \mathscr{L}_{Y_{2}}} Y_{1}+Y_{2}^{*} \mathscr{R}_{Y_{2} \mathscr{L}_{Y_{1}}} S_{2} \mathscr{R}_{Y_{2} \mathscr{L}_{Y_{1}}} Y_{2}=G
$$

Thus we need to obtain a pair of Hermitian solutions $\left(\widehat{S}_{1}, \widehat{S}_{2}\right)$ of the linear matrix equation (3.11). Firstly, we give the generalized singular value decomposition (GSVD) of the matrix pair $\left(\mathscr{R}_{Y_{1} \mathscr{L}_{Y_{2}}} Y_{1}, \mathscr{R}_{Y_{2} \mathscr{L}_{Y_{1}}} Y_{2}\right)$ as follows (see, for example, [12]):

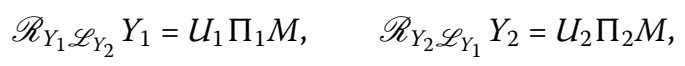

where $U_{1}$ and $U_{2}$ are unitary matrices of order $k$ and $M \in \mathbb{C}^{m \times m}$ is a nonsingular matrix, and

$$
\begin{aligned}
& \Pi_{1}=\left(\begin{array}{cccc}
I_{r_{3}-r_{2}} & \mathbf{0} & \mathbf{0} & \mathbf{0} \\
\mathbf{0} & \Lambda_{1} & \mathbf{0} & \mathbf{0} \\
\mathbf{0} & \mathbf{0} & \mathbf{0} & \mathbf{0}
\end{array}\right) \begin{array}{l}
r_{3}-r_{2} \\
r_{1}+r_{2}-r_{3} \\
k-r_{1}
\end{array}, \\
& r_{3}-r_{2} \quad r_{1}+r_{2}-r_{3} \quad r_{3}-r_{1} \quad m-r_{3}
\end{aligned}
$$

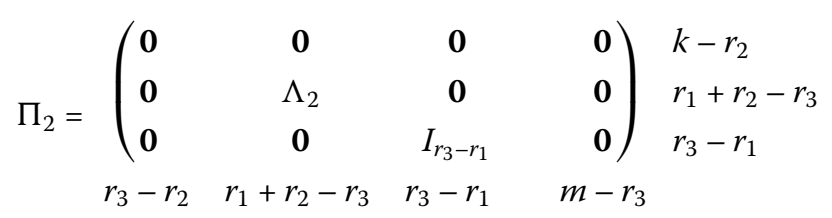

are block matrices with the same column partitioning. In the matrices $\Pi_{1}$ and $\Pi_{2}$,

$$
\begin{aligned}
& r_{1}=\operatorname{rank}\left(\mathscr{R}_{Y_{1} \mathscr{L}_{Y_{2}}} Y_{1}\right), \quad r_{2}=\operatorname{rank}\left(\mathscr{R}_{Y_{2} \mathscr{L}_{Y_{1}}} Y_{2}\right), \\
& r_{3}=\operatorname{rank}\left(Y_{1}^{*} \mathscr{R}_{Y_{1} \mathscr{L}_{Y_{2}}}, Y_{2}^{*} \mathscr{R}_{Y_{2} \mathscr{L}_{Y_{1}}}\right), \\
& \Lambda_{1}=\operatorname{diag}\left(\xi_{1}, \xi_{2}, \ldots, \xi_{r_{1}+r_{2}-r_{3}}\right), \quad 1>\xi_{1} \geq \cdots \geq \xi_{r_{1}+r_{2}-r_{3}}>0,
\end{aligned}
$$




$$
\begin{aligned}
& \Lambda_{2}=\operatorname{diag}\left(\eta_{1}, \eta_{2}, \ldots, \eta_{r_{1}+r_{2}-r_{3}}\right), \quad 0<\eta_{1} \leq \cdots \leq \eta_{r_{1}+r_{2}-r_{3}}<1, \\
& \Lambda_{1}^{2}+\Lambda_{2}^{2}=I_{r_{1}+r_{2}-r_{3}} .
\end{aligned}
$$

We further partition the nonsingular matrix

$$
M^{-1}=\left(\begin{array}{cccc}
M_{1} & M_{2} & M_{3} & M_{4} \\
r_{3}-r_{2} & r_{1}+r_{2}-r_{3} & r_{3}-r_{1} & m-r_{3}
\end{array}\right)
$$

compatibly with the block column partitioning of $\Pi_{1}$ or $\Pi_{2}$. Denote

$$
\left(M^{*}\right)^{-1} G M^{-1}=\left(G_{i j}\right)_{4 \times 4} \quad \text { with } G_{i j}=M_{i}^{*} G M_{j}, i, j=1,2,3,4 \text {. }
$$

Then substitute (3.12) into (3.11). By [13, Theorem 3.1] we obtain that equation (3.11) is consistent if and only if

$$
\begin{aligned}
& Y_{1}^{*} Y_{1} \Lambda+\Lambda^{*} Y_{2}^{*} Y_{2}=\Lambda^{*} Y_{1}^{*} Y_{1}+Y_{2}^{*} Y_{2} \Lambda, \\
& G_{13}=0, \quad G_{14}=0, \quad G_{24}=0, \quad G_{34}=0, \quad G_{44}=0 .
\end{aligned}
$$

Moreover, its general solution can be expressed as

$$
\begin{aligned}
& \widehat{S}_{1}=U_{1}\left[\begin{array}{ccc}
G_{11} & G_{12} \Lambda_{1}^{-1} & X_{13} \\
\Lambda_{1}^{-1} G_{12}^{*} & \Lambda_{1}^{-1}\left(G_{22}-\Lambda_{2} Y_{22} \Lambda_{2}\right) \Lambda_{1}^{-1} & X_{23} \\
X_{13}^{*} & X_{23}^{*} & X_{33}
\end{array}\right] U_{1}^{*}, \\
& \widehat{S}_{2}=U_{2}\left[\begin{array}{ccc}
Y_{11} & Y_{12} & Y_{13} \\
Y_{12}^{*} & Y_{22} & \Lambda_{2}^{-1} G_{23} \\
Y_{13}^{*} & G_{23}^{*} \Lambda_{2}^{-1} & G_{33}
\end{array}\right] U_{2}^{*},
\end{aligned}
$$

where $X_{33}, Y_{11}$, and $Y_{22}$ are arbitrary Hermitian matrices, $X_{13}, X_{23}, Y_{12}$, and $Y_{13}$ are arbitrary matrices.

Then substituting (3.15) and (3.16) into (3.7) and (3.9) yields

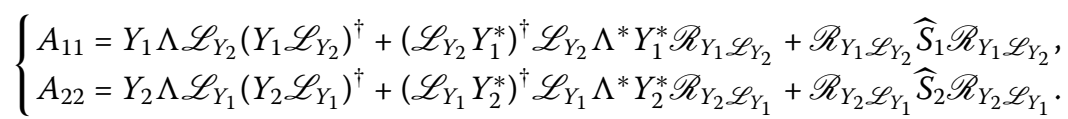

From (3.4), (3.17), and Lemma 3, we get

$$
\begin{aligned}
A_{12}= & \left(Y_{1}^{*}\right)^{\dagger}\left(Y_{2}^{*} A_{22}-\Lambda^{*} Y_{2}^{*}\right)+\mathscr{R}_{Y_{1}}\left(Y_{1} \Lambda-A_{11} Y_{1}\right)\left(Y_{2}^{*}\right)^{\dagger}+\mathscr{R}_{Y_{1}} R \mathscr{R}_{Y_{2}} \\
= & \left(Y_{1}^{\dagger}\right)^{*} Y_{2}^{*} Y_{2} \Lambda \mathscr{L}_{Y_{1}}\left(Y_{2} \mathscr{L}_{Y_{1}}\right)^{\dagger}+\left(Y_{1}^{\dagger}\right)^{*} Y_{2}^{*}\left(\mathscr{L}_{Y_{1}} Y_{2}^{*}\right)^{\dagger} \mathscr{L}_{Y_{1}} \Lambda^{*} Y_{2}^{*} \mathscr{R}_{Y_{2} \mathscr{L}_{Y_{1}}} \\
& -\mathscr{R}_{Y_{1}}\left(\mathscr{L}_{Y_{2}} Y_{1}^{*}\right)^{\dagger} \mathscr{L}_{Y_{2}} \Lambda^{*} Y_{1}^{*} \mathscr{R}_{Y_{1}} \mathscr{L}_{Y_{2}} Y_{1} Y_{2}^{\dagger}-\left(Y_{1}^{\dagger}\right)^{*} \Lambda^{*} Y_{2}^{*} \\
& +\left(Y_{1}^{\dagger}\right)^{*} Y_{2}^{*} \mathscr{R}_{Y_{2}} \mathscr{L}_{Y_{1}} \widehat{S}_{2} \mathscr{R}_{Y_{2}} \mathscr{L}_{Y_{1}}-\mathscr{R}_{Y_{1}} \mathscr{R}_{Y_{1}} \mathscr{L}_{Y_{2}} \widehat{S}_{1} \mathscr{R}_{Y_{1}} \mathscr{L}_{Y_{2}} Y_{1} Y_{2}^{\dagger} \\
& +\mathscr{R}_{Y_{1}} R \mathscr{R}_{Y_{2}},
\end{aligned}
$$

where $R \in \mathbb{C}^{k \times k}$ is an arbitrary matrix.

Based on the above discussion, we can conclude the following result to solve Problem 1. 
Theorem 1 Given $Y \in \mathbb{C}^{n \times m}$ and $\Lambda \in \mathbb{C}^{m \times m}$ as described in Problem 1. Let $U^{*} Y, G$, the

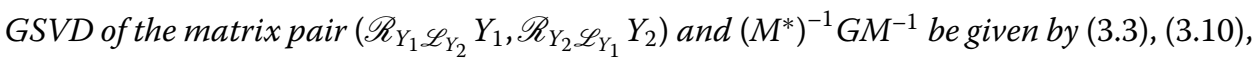
(3.12), and (3.13), respectively. Then Problem 1 is solvable(i.e., $\mathcal{M}(Y, \Lambda) \neq \emptyset)$ in the set $\mathcal{N S}^{n \times n}(J)$ if and only if conditions (3.6), (3.8), (3.14), and $A_{11} A_{12}=A_{12} A_{22}$ hold. Moreover, in this case, the general solution can be expressed as

$$
A=U\left[\begin{array}{cc}
A_{11} & A_{12} \\
-A_{12}^{*} & A_{22}
\end{array}\right] U^{*}
$$

where $A_{11}, A_{22}$, and $A_{12}$ are given by (3.17) and (3.18), respectively. In the matrices $A_{11}$, $A_{22}$, and $A_{12}, \widehat{S}_{1}$ and $\widehat{S}_{2}$ are described in (3.15) and (3.16), respectively, where $X_{33}, Y_{11}$, and $Y_{22}$ are arbitrary Hermitian matrix blocks, $X_{13}, X_{23}, Y_{12}, Y_{13}$, and $R$ are arbitrary matrix blocks.

\section{Conclusions}

In this paper, we have obtained the necessary and sufficient conditions of the inverse eigenvalue problem for normal skew $J$-Hamiltonian matrices. Furthermore, a solvable general representation is presented. We can also use the same method to solve the inverse eigenvalue problem for a normal $J$-Hamiltonian matrix.

Acknowledgements

Not applicable.

Funding

Not applicable.

Abbreviations

Not applicable.

Availability of data and materials

Not applicable.

Competing interests

The authors declare that they have no competing interests.

Authors' contributions

The authors declare that the study was realized in collaboration with the same responsibility. All authors read and approved the final manuscript.

\section{Publisher's Note}

Springer Nature remains neutral with regard to jurisdictional claims in published maps and institutional affiliations.

Received: 30 October 2017 Accepted: 28 March 2018 Published online: 04 April 2018

\section{References}

1. Gigola, S., Lebtahi, L., Thome, N.: Inverse eigenvalue problem for normal J-Hamiltonian matrices. Appl. Math. Lett. 48, 36-40 (2015)

2. Chu, M.T., Golub, G.H.: Inverse Eigenvalue Problems: Theory, Algorithms, and Application. Oxford University Press, Oxford (2005)

3. Cox, S.J., Embree, M., Hokanson, J.M.: One can hear the composition of a string: experiments with an inverse eigenvalue problem. SIAM Rev. 54, 157-178 (2012)

4. Hu, S.-L.J., Li, H.: A systematic linear space approach to solving partially described inverse eigenvalue problems. Inverse Probl. 24, 035014 (2008)

5. Zhang, Z., Hu, X., Zhang, L.: The solvability conditions for the inverse eigenvalue problem of Hermitian-generalized Hamiltonian matrices. Inverse Probl. 18, 1369-1376 (2002)

6. Bai, Z.: The solvability conditions for the inverse eigenvalue problem of Hermitian and generalized skew-Hamiltonian matrices and its approximation. Inverse Probl. 19, 1185-1194 (2003)

7. Xie, D., Huang, N., Zhang, Q.: An inverse eigenvalue problem and a matrix approximation problem for symmetric skew-Hamiltonian matrices. Numer. Algorithms 46, 23-34 (2007) 
8. Qian, J., Tan, R.C.E.: On some inverse eigenvalue problems for Hermitian and generalized Hamiltonian/skew-Hamiltonian matrices. J. Comput. Appl. Math. 250, 28-38 (2013)

9. Jamshidi, M.: An overview on the solutions of the algebraic matrix Riccati equation and related problems. In: Large Scale Systems, Theory and Applications, vol. 1, pp. 167-192 (1980)

10. Sun, J.: Two kinds of inverse eigenvalue problems for real symmetric matrices. Math. Numer. Sin. 3, $282-290$ (1988)

11. Penrose, R.: A generalized inverse for matrices. Proc. Camb. Philos. Soc. 51, 406-413 (1955)

12. Herrero, A., Thome, N.: Using the GSVD and the lifting technique to find $\{P, k+1\}$ reflexive and anti-reflexive solutions of $A X B=$ C. Appl. Math. Lett. 24, 1130-1141 (2011)

13. Chang, $X$., Wang, J.: The symmetric solution of the matrix equations $A X+Y A=C, A X A^{T}+B Y B^{T}=C$, and $\left(A^{\top} X A, B^{T} X B\right)=(C, D)$. Linear Algebra Appl. 179, 171-189(1993)

\section{Submit your manuscript to a SpringerOpen ${ }^{\circ}$} journal and benefit from:

- Convenient online submission

- Rigorous peer review

- Open access: articles freely available online

- High visibility within the field

- Retaining the copyright to your article

Submit your next manuscript at $\gg$ springeropen.com 